\title{
On Statistical Development of Neutrosophic Gamma Distribution with Applications to Complex Data Analysis
}

\author{
Zahid Khan $\mathbb{D},{ }^{1}$ Afrah Al-Bossly, ${ }^{2}$ Mohammed M. A. Almazah $\mathbb{D},{ }^{3,4}$ and Fuad S. Alduais $\mathbb{D}^{2,5}$ \\ ${ }^{1}$ Department of Mathematics and Statistics, Hazara University, Mansehra, Pakistan \\ ${ }^{2}$ Department of Mathematics, College of Science and Humanities in Al-Kharj, Prince Sattam Bin Abdulaziz University, \\ Al-Kharj 11942, Saudi Arabia \\ ${ }^{3}$ Department of Mathematics, College of Sciences and Arts (Muhyil), King Khalid University, Muhyil, Abha, Asir Region 61421, \\ Saudi Arabia \\ ${ }^{4}$ Department of Mathematics and Computer, College of Sciences, Ibb University, Ibb 70270, Yemen \\ ${ }^{5}$ Country Business Administration Department, Administrative Science College, Thamar University, Thamar, Yemen
}

Correspondence should be addressed to Zahid Khan; zahidkhan@hu.edu.pk

Received 18 September 2021; Revised 1 December 2021; Accepted 3 December 2021; Published 18 December 2021

Academic Editor: Zeljko Stevic

Copyright (c) 2021 Zahid Khan et al. This is an open access article distributed under the Creative Commons Attribution License, which permits unrestricted use, distribution, and reproduction in any medium, provided the original work is properly cited.

In the absence of a correct distribution theory for complex data, neutrosophic algebra can be very useful in quantifying uncertainty. In applied data analysis, implementation of existing gamma distribution becomes inadequate for some applications when dealing with an imprecise, uncertain, or vague dataset. Most existing works have explored distributional properties of the gamma distribution under the assumption that data do not have any kind of indeterminacy. Yet, analytical properties of the gamma model for the more realistic setting when data involved uncertainties remain largely underdeveloped. This paper fills such a gap and develops the notion of neutrosophic gamma distribution (NGD). The proposed distribution represents a generalized structure of the existing gamma distribution. The basic distributional properties, including moments, shape coefficients, and moment generating function (MGF), are established. Several examples are considered to emphasize the relevance of the proposed NGD for dealing with circumstances with inadequate or ambiguous knowledge about the distributional characteristics. The estimation framework for treating vague parameters of the NGD is developed. The Monte Carlo simulation is implemented to examine the performance of the proposed model. The proposed model is applied to a real dataset for the purpose of dealing with inaccurate and vague statistical data. Results show that the NGD has better flexibility in handling real data over the conventional gamma distribution.

\section{Introduction}

The gamma distribution is named after the widely known gamma function (GF), which is explored in many fields of mathematics. The gamma distribution in applied statistical analysis is used extensively for studying stochastic phenomena [1]. Wang and $\mathrm{Wu}$ [2] presented valuable information on the gamma distribution and its uses. Three types of gamma distribution functions, namely, one, two, and three parameter gamma densities, are employed as wellsuited models for many real datasets [3-5]. The gamma distribution family portrays three different failure rates in survival and reliability analysis [6]. This resulted in the gamma model being reasonably versatile and describing various lifespan data [7]. It has been frequently applied in numerous disciplines, including metagenomics [8], hydrology [9], environmental science [10], climatology [11], and genetics [12]. Some excellent work can be found in the inference part of the gamma distribution in $[13,14]$. Due to extensive applications of the gamma distribution, many generalized structures have been suggested in the literature $[15,16]$. The basic model and generalized structures of the gamma distribution are rooted in the assumption that distributional parameters accurately describe a particular set of data, which is unrealistic in many situations. For example, the scale and shape parameters of the gamma density cannot 
be fixed to exactly single numbers to find a well-suited model for the amount of rainfall data. However, it is more realistic to believe that the gamma model is a best-described distribution for the rainfall data with an interval set of values for shape and scale parameters. The notion of the neutrosophy can model such indeterminacies in the data originated by Smarandache [17]. It is a new philosophical area, an extension for fuzzy and intuitionistic fuzzy logics [18-22]. Smarandache suggested the essential principles of neutrosophic set in [23-27]. Classical statistics deals with determinate data and determinate inference methods only, neutrosophic statistics deals with indeterminate data and indeterminate inference methods, i.e., data that have any kind of indeterminacy (unclear, vague, partially unknown, contradictory, incomplete, etc.), and inference methods that have degrees of indeterminacy as well (for example, instead of crisp arguments and values for the probability distributions, algorithms, functions etc., one may have inexact or ambiguous arguments and values). Neutrosophic statistics was founded by Smarandache in 1998 and developed in 2014 [24]. The neutrosophic statistics is also a generalization of interval statistics because, among others, interval statistics is based on interval analysis. Neutrosophic statistics is based on set analysis (meaning all kinds of sets, not only intervals). Neutrosophic statistics is more elastic than classical statistics. If all data and inference methods are determinate, then neutrosophic statistics coincides with classical statistics. But, since, in our world, we have more indeterminate data than determinate data, more neutrosophic statistical procedures are needed than classical ones. Because the parameters of classical gamma distribution are indeterminate in the neutrosophic logic, it is possible to deal with all the scenarios that one may find while working with real data analysis [28-34]. Recently, Duan et al. [35] introduced the algebraic structure of the exponential distribution. Sherwani et al. [36] described the neutrosophic version of the normal distribution. More applications of the neutrosophic statistics can be seen in [37-40].

In the interest of more practical data modelling, this study proposes a new extension of gamma distribution under neutrosophic logic. Some research work exists on neutrosophic distributions, for example, [34], but we expect that the current study may be complete by presenting a comprehensive overview of NGD and providing more insights on it. The purpose of the present study is to introduce a neutrosophic version of the gamma distribution. Such an extension is potentially relevant for practical applications since there are far fewer distributions available for dealing with indeterminate data in the univariate and multivariate cases, especially for situations where data are not precise and reported in fuzzy or interval forms.

The rest of the work is described as follows: The NGD is presented in Section 2. Section 3 provides some examples for conceptual understanding of the NGD. The distributional parameters are estimated in Section 4. Finally, a real data analysis using NGD is explained in Section 5. Lastly, Section 6 concludes the findings of the study.

\section{Neutrosophic Gamma Model}

The random variable $Z$ is neutrosophic gamma distributed with parameters $p_{n}>0$ and $\lambda_{n}>0$ if it has the following probability function:

$$
\xi_{n}(z)=\frac{1}{\Gamma p_{n} \lambda_{n}^{p_{n}}} z^{p_{n}-1} e^{-\left(z / \lambda_{n}\right)}, \quad z>0,
$$

where $\Gamma$ is well-known GF and for a neutrosophic value, $\Gamma p_{n}=\int_{0}^{\infty} t^{p_{n}-1} e^{-t} \mathrm{~d} t$.

$\mathrm{Z}$ follows the neutrosophic Erlang model for integer values of $p_{n}$. The distributional parameters $p_{n}$ and $\left(1 / \lambda_{n}\right)$ are frequently recognized as the shape and scale parameters, respectively. Sketches of the NGD for several values of $p_{n}$ and $\lambda_{\mathrm{n}}$ are shown in Figures 1 and 2.

In Figures 1 and 2, parameters settings may be changed to create various neutrosophic gamma curves. In Figure 1, the shape parameter is fixed, i.e., $p_{n}=[8,8]$, while the scale parameter $\lambda_{n}$ varies to three different values. However, in Figure 2, the scale parameter is fixed to a crisp value $[6,6]$ but shape parameter $p_{n}$ varies to three different values. The area under a particular neutrosophic curve is always equal to one that can easily be shown by using the transformation $y=\left(z / \lambda_{n}\right)$ in (1). Equation (1) can be used to compute the probability for a neutrosophic gamma random variable. Nevertheless, it is difficult to calculate the integral of the NGD using a mathematical expression, so probabilities are evaluated using computer software. These may be evaluated via repeated integration by parts, but the details are long and ignored. Some useful distributional properties of NGD can be described in the forms of some theorems as follows.

Theorem 1. The mean of the NGD is $p_{n} \lambda_{n}$.

Proof. Using the definition of expected value, mean of the NGD can be written as

$$
\mu_{n}=\int_{0}^{\infty} \frac{z}{\Gamma p_{n} \lambda_{n}^{p_{n}}} z^{p_{n}-1} e^{-\left(z / \lambda_{n}\right)} \mathrm{d} z
$$

Using the neutrosophic calculus, expression (2) can be further simplified as

$$
\begin{aligned}
& \mu_{n}=\int_{0}^{\infty} z\left[\frac{1}{\Gamma p_{l} \lambda_{l}^{p_{l}}} z^{p_{l}-1} e^{-\left(z / \lambda_{l}\right)}, \frac{1}{\Gamma p_{u} \lambda_{u}^{p_{u}}} z^{p_{u}-1} e^{-\left(z / \lambda_{u}\right)}\right] \mathrm{d} z, \\
& \mu_{n}=\left[\int_{0}^{\infty} \frac{z}{\Gamma p_{l} \lambda_{l}^{p_{l}}} z^{p_{l}-1} e^{-\left(z / \lambda_{l}\right)} \mathrm{d} z, \int_{0}^{\infty} \frac{z}{\Gamma p_{u} \lambda_{u}^{p_{u}}} z^{p_{u}-1} e^{-\left(z / \lambda_{u}\right)} \mathrm{d} z\right] .
\end{aligned}
$$

Substituting $y=\left(z / \lambda_{n}\right)$ in (3) yielded

$$
\begin{gathered}
\int_{0}^{\infty} \frac{z}{\Gamma p_{l} \lambda_{l}^{p_{l}}} z^{p_{l}-1} e^{-\left(z / \lambda_{l}\right)} \mathrm{d} z=p_{l} \lambda_{l}, \\
\int_{0}^{\infty} \frac{z}{\Gamma p_{u} \lambda_{u}^{p_{u}}} z^{p_{u}-1} e^{-\left(z / \lambda_{u}\right)} \mathrm{d} z=p_{u} \lambda_{u} .
\end{gathered}
$$

Thus, (3) provides 


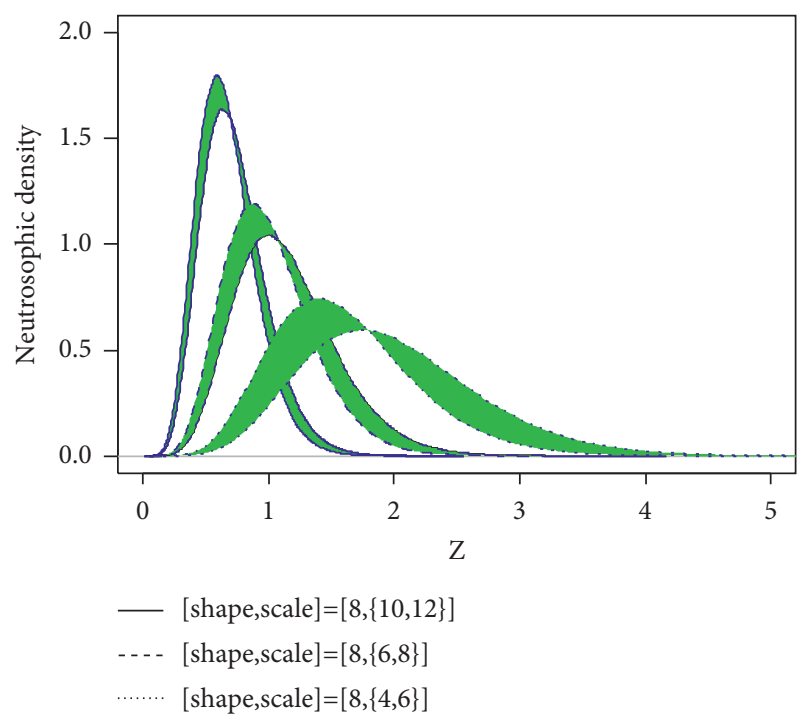

FIgURE 1: The density of the NGD with the neutrosophic scale parameter.

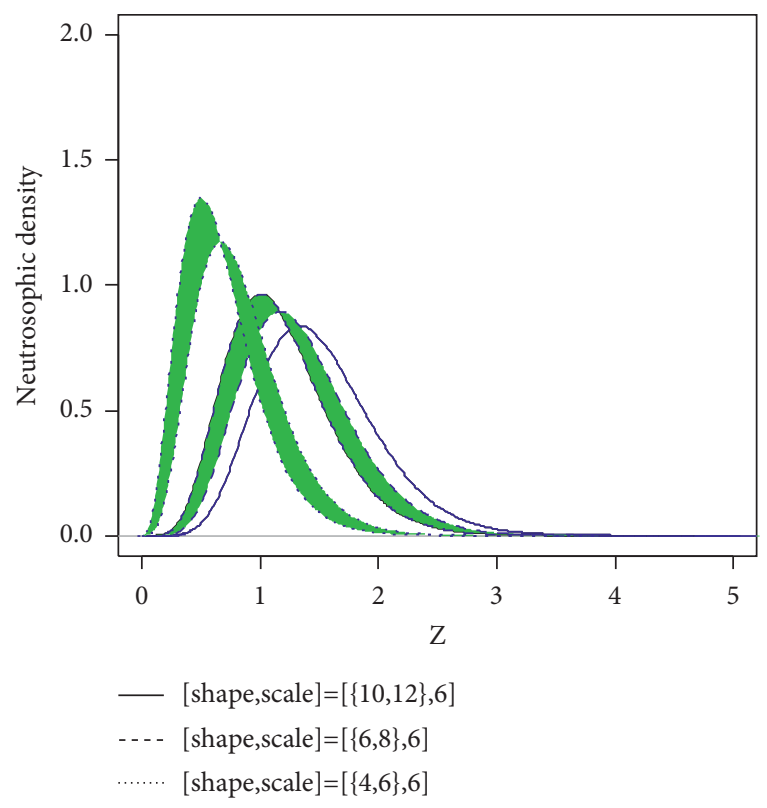

FIgURE 2: The density of the NGD with the neutrosophic shape parameter.

$$
\mu_{n}=\left[p_{l} \lambda_{l}, p_{u} \lambda_{u}\right]=p_{n} \lambda_{n} .
$$

Hence proved.

Theorem 2. The variance of NGD is $p_{n} \lambda_{n}^{2}$.

Proof. By definition, variance of NGD is given by

$$
\sigma_{n}^{2}=E\left(Z^{2}\right)-[E(Z)]^{2}
$$

Now,

$$
\begin{aligned}
E\left(Z^{2}\right) & =\int_{0}^{\infty} \frac{z^{2}}{\Gamma p_{n} \lambda_{n}^{p_{n}}} z^{p_{n}-1} e^{-\left(z / \lambda_{n}\right)} \mathrm{d} z \\
& =\left[\int_{0}^{\infty} \frac{\omega_{l} z^{2}}{\Gamma p_{l} \lambda_{l}^{p_{l}}} z^{p_{l}-1} e^{-\left(z / \lambda_{l}\right)} \mathrm{d} z, \int_{0}^{\infty} \frac{\omega_{u} z^{2}}{\Gamma p_{u} \lambda_{u}^{p_{u}}} z^{p_{u}-1} e^{-\left(z / \lambda_{u}\right)} \mathrm{d} z\right] .
\end{aligned}
$$

From (7), simplification provided

$$
\begin{gathered}
\int_{0}^{\infty} \frac{\omega_{l} z^{2}}{\Gamma p_{l} \lambda_{l}^{\alpha_{l}}} z^{p_{l}-1} e^{-\left(z / \lambda_{l}\right)} \mathrm{d} z=\lambda_{l}^{2} p_{l}\left(p_{l}+1\right), \\
\int_{0}^{\infty} \frac{\omega_{u} z^{2}}{\Gamma p_{u} \lambda_{u}^{p_{u}}} z^{p_{u}-1} e^{-\left(z / \lambda_{u}\right)} \mathrm{d} z=\lambda_{u}^{2} p_{u}\left(p_{u}+1\right) .
\end{gathered}
$$

Thus, (7) becomes

$$
E\left(Z^{2}\right)=\left[\lambda_{l}^{2} p_{l}\left(p_{l}+1\right), \lambda_{u}^{2} p_{u}\left(p_{u}+1\right)\right] .
$$

Substituting (5) and (9) in (6) yielded $\sigma_{n}^{2}=\left[p_{l} \lambda_{l}^{2}, p_{u} \lambda_{u}^{2}\right]$ $=p_{n} \lambda_{n}^{2}$; hence proved.

Theorem 3. The $r^{\text {th }}$ moment of NGD is $\lambda_{n}^{r} / \Gamma p_{n}\left(\Gamma r+p_{n}\right)$.

Proof. By definition, the $j^{\text {th }}$ moment of NGD is given by

$$
\begin{aligned}
\mu_{j n}^{\prime} & =\int_{0}^{\infty} \frac{z^{j}}{\Gamma p_{n} \lambda_{n}^{p_{n}}} z^{p_{n}-1} e^{-\left(z / \lambda_{n}\right)} \mathrm{d} z \\
& =\int_{0}^{\infty} z^{j}\left[\frac{1}{\Gamma p_{l} \lambda_{l}^{p_{l}}} z^{p_{l}-1} e^{-\left(z / \lambda_{l}\right)}, \frac{1}{\Gamma p_{u} \lambda_{u}^{p_{u}}} z^{p_{u}-1} e^{-\left(z / \lambda_{u}\right)}\right] \mathrm{d} z \\
& =\left[\int_{0}^{\infty} z^{j} \frac{1}{\Gamma p_{l} \lambda_{l}^{p_{l}}} z^{p_{l}-1} e^{-\left(z / \lambda_{l}\right)} \mathrm{d} z, \int_{0}^{\infty} z^{j} \frac{1}{\Gamma p_{u} \lambda_{u}^{p_{u}}} z^{p_{u}-1} e^{-\left(z / \lambda_{u}\right)} \mathrm{d} z\right] .
\end{aligned}
$$

Substituting $y=\left(z / \lambda_{n}\right)$ in (10) yielded

$$
\begin{gathered}
\int_{0}^{\infty} z^{j} \frac{\omega_{l}}{\Gamma p_{l} \lambda_{l}^{p_{l}}} z^{p-1} e^{-\left(z / \lambda_{l}\right)} \mathrm{d} z=\frac{\lambda_{l}^{j}}{\Gamma p_{l}}\left(\Gamma j+p_{l}\right), \\
\int_{0}^{\infty} z^{j} \frac{\omega_{u}}{\Gamma p_{u} \lambda_{u}^{p_{u}}} z^{p_{u}-1} e^{-\left(z / \lambda_{u}\right)} \mathrm{d} z=\frac{\lambda_{u}^{j}}{\Gamma p_{u}}\left(\Gamma j+p_{u}\right) .
\end{gathered}
$$

Thus, (10) becomes

$$
\mu_{j n}^{\prime}=\left[\frac{\lambda_{l}^{j}}{\Gamma p_{l}}\left(\Gamma j+p_{l}\right), \frac{\lambda_{u}^{j}}{\Gamma p_{u}}\left(\Gamma j+p_{u}\right)\right]=\frac{\lambda_{n}^{j}}{\Gamma p_{n}} ; \quad j=1,2, \ldots,
$$

where $\mu_{j n}^{\prime}$ is the $j$ th moment about origin. However, moments about mean can be established by using the wellknown relation as

$$
\begin{aligned}
& \mu_{1 n}=\lambda_{n}, \\
& \mu_{2 n}=\lambda_{n}, \\
& \mu_{3 n}=2 \lambda_{n}, \\
& \mu_{4 n}=6 \lambda_{n}-3 \lambda_{n}^{2} .
\end{aligned}
$$


Theorem 4. The coefficient of skewness of NGD is shown as $\left(2 / \sqrt{\lambda_{n}}\right)$.

Proof. By definition, the skewness of the NGD is given by

$$
\gamma_{1 n}=\frac{\mu_{3 N}}{\left(\mu_{2 n}\right)^{3 / 2}},
$$

where $\mu_{3 N}=2 \lambda_{n}$ and $\mu_{2 N}=\lambda_{n}$.

Simplifying (7) further provides

$$
\gamma_{1 n}=\frac{2}{\sqrt{\lambda_{n}}}
$$

where $\gamma_{1 n} \in\left[\gamma_{1 l}, \gamma_{1 u}\right]$.

This coefficient is in a standardized form and provides information about the spread of the distribution around its mean value. The standardized form is unit free and of much help to compare two variables computed in a different unit of measurements. The NGD curve in Figure 1 exhibits positive skew due to the positive value of skewness.

Theorem 5. The coefficient of kurtosis of the NGD is shown as $\left(6 / \lambda_{n}\right)-3$.

Proof. The coefficient of kurtosis of the distribution is given by

$$
\gamma_{2 n}=\frac{\mu_{4 N}}{\left(\mu_{2 n}\right)^{2}}-3
$$

From Theorem 3, it follows that $\mu_{2 n}=\lambda_{n}$ and $\mu_{4 n}=6 \lambda_{n}-3 \lambda_{n}^{2}$.

Simplification of (7) implies $\gamma_{2 n}=\left(6 / \lambda_{n}\right)-3$, which is the required result.

The kurtosis coefficient is also in the standardized form and provides information about the spread of the distribution. It is more related to extreme points of the distribution.

Theorem 6. The MGF of the NGD is $1 /\left(1-t p_{n}\right)^{\lambda_{n}}$.

Proof. The MGF of the NGD is given by

$$
\begin{aligned}
M_{n}(t) & =\int_{0}^{\infty} \frac{e^{t Z}}{\Gamma p_{n} \lambda_{n}^{p_{n}}} z^{p_{n}-1} e^{-\left(z / \lambda_{n}\right)} \mathrm{d} z \\
& =\int_{0}^{\infty}\left[\frac{1}{\Gamma p_{l} \lambda_{l}^{p_{l}}} z^{p_{l}-1} e^{-z\left(1 / \lambda_{n}-t\right)}, \frac{1}{\Gamma p_{u} \lambda_{u}^{p_{u}}} z^{p_{u}-1} e^{-z\left(1 / \lambda_{n}-t\right)}\right] \mathrm{d} z .
\end{aligned}
$$

Using the transformation $y_{l}=z\left(1 / \lambda_{l}-t\right)$ and $y_{u}=z\left(1 / \lambda_{u}-t\right)$ in (17), simplification resulted

$$
\begin{aligned}
& =\left[\frac{1}{\Gamma p_{l} \lambda_{l}^{p_{l}}}\left(\frac{\lambda_{l}}{1-\lambda_{l} t}\right)^{p_{l}} \int_{o}^{\infty} y_{l}^{p_{n}-1} e^{-y_{l}} \mathrm{~d} y_{l}, \frac{1}{\Gamma p_{u} \lambda_{u}^{u}}\left(\frac{\lambda_{u}}{1-\lambda_{u} t}\right)^{p_{u}} \int_{o}^{\infty} y_{l}^{p_{u}-1} e^{-y_{u}} \mathrm{~d} y_{u}\right] \\
& =\left[\left(\frac{1}{1-\lambda_{l} t}\right)^{p_{l}},\left(\frac{1}{1-\lambda_{u} t}\right)^{p_{u}}\right] .
\end{aligned}
$$

Thus,

$$
M_{n}(t)=\left(\frac{1}{1-\lambda_{n} t}\right)^{p_{n}}
$$

The expression given in (19) can be used to generate moments of the NGD discussed in Theorem 3.

\section{Sample Estimation}

The method of maximum likelihood is commonly recommended to find the known values of conventional parameters of the gamma distribution. Let $z_{1}, z_{2}, \ldots, z_{m}$ be a random sample from the NGD, and $\varphi\left(p_{n}, \lambda_{n} \mid z\right)$ denotes the $\log$ likelihood function; then,

$$
\begin{aligned}
\varphi\left(p_{n}, \lambda_{n} \mid z\right)= & \left(p_{n}-1\right) \sum_{i=1}^{m} \ln \left(z_{i}\right)-\frac{\sum_{i=1}^{m} z_{i}}{\lambda_{n}} \\
& -m \ln \left(\Gamma p_{n}\right)-m p_{n} \log \left(\lambda_{n}\right) .
\end{aligned}
$$

Differentiating (20) with respect to $\lambda_{n}$ and $p_{n}$ yielded

$$
\begin{aligned}
& \frac{\delta \varphi\left(p_{n}, \lambda_{n} \mid z\right)}{\delta \lambda_{n}}=\frac{\sum_{i=1}^{m} z_{i}}{\lambda_{n}^{2}}-\frac{m p_{n}}{\lambda_{n}}, \\
& \frac{\delta \varphi\left(p_{n}, \lambda_{n} \mid z\right)}{\delta \lambda_{n}}=n\left[\frac{\sum_{i=1}^{n} \ln \left(z_{i}\right)}{n}-\ln \left(\lambda_{n}\right)-\omega(p)\right],
\end{aligned}
$$

where $\omega(p)=\left(\mathrm{d} \ln \left(p_{n}\right) / \mathrm{d} p_{n}\right)$ is known as the digamma function.

Now, equating (21) and (22) to zero and simultaneous solution for $p_{n}$ and $\lambda_{n}$ provide

$$
\begin{array}{r}
\lambda_{n}=\frac{\bar{z}}{p_{n}}, \\
\overline{\ln (z)}-\ln (\bar{z})+\ln \left(p_{n}\right)-\omega(p)=0 .
\end{array}
$$

From (23) and (24), maximum values of $p_{n}$ and $\lambda_{n}$ can be achieved by using an iterative numerical algorithm. $\mathrm{R}$ software is more convenient to perform this algorithm. To assess the performance of distributional estimators in terms of neutrosophic average biased $\left(\mathrm{AB}_{n}\right)$ and neutrosophic root measure square error $\left(\mathrm{RME}_{n}\right)$, a simulation study has also been conducted. Assessment measures $\mathrm{AB}_{n}$ and $\mathrm{RME}_{n}$ are defined as follows [41]: 


$$
\begin{aligned}
\mathrm{AB}_{n} & =\frac{\sum_{i=1}^{N}\left(\hat{\vartheta}_{n i}-\vartheta_{n}\right)}{N}, \\
\mathrm{RME}_{n} & =\sqrt{\frac{\sum_{i=1}^{N}\left(\hat{\vartheta}_{n i}-\vartheta_{n}\right)^{2}}{N}},
\end{aligned}
$$

where $\vartheta_{n}$ is the shape or scale parameter and $\widehat{\vartheta}_{n}$ is a corresponding estimate of the NGD.

Using the $\mathrm{R}$ programming language, a Monte Carlo simulation is performed with different sample sizes and fixed values of the neutrosophic parameters $\lambda_{n}=[5,8]$ and $p_{n}=[1,1]$. An imprecise dataset is produced with defined parametric values, and simulation analysis is replicated $N=$ $10^{5}$ times with sample sizes of $m=5,15,30$, and 60 , respectively. The performance metrics of the distributional estimators are then estimated and listed in Table 1.

To evaluate the reliability of maximum likelihood estimators, $\mathrm{AB}_{n}$ and $\mathrm{RME}_{n}$ are computed over the given sample size. While the metrics used provide a numerical evaluation of estimation accuracy, they are difficult to assess without a baseline value. The parameter setting $\lambda_{n}=[5,8]$ and $p_{n}=$ $[1,1]$ are used as baseline values for the evaluation of estimation accuracy. The results show that when the sample size $n$ increases, $\mathrm{AB}_{n}$, i.e., biases, decrease. On this account, the study shows that with a higher sample size, the neutrosophic estimators provide reliable estimates. Thus, the differences between the baseline values and estimated values are decreased when the sample size increases.

\section{Illustrative Examples}

Some illustrative examples are given in this section to understand the theoretical results presented in the previous section.

Example 1. In a biomedical investigation using rats, a medicate-response experiment is performed to evaluate the influence of a toxicant's dosage on their survival time. This toxic substance is one which is ordinarily released into the atmosphere as a byproduct of jet fuel. The study indicates that for a given toxicant dosage, the survival time, in weeks, has an NGD with $p_{n}=[4,6]$ and $\lambda_{n}=1$. What is the probability that rats will survive for no more than 6 weeks? Also, find the mean survival time of randomly selected rats in this experiment.

Solution: let the random variable $Z$ denotes the survival times of rats in weeks.

Then, using (1), we can write

$$
P(Z \leq 6)=\int_{0}^{6} \frac{1}{\Gamma[4,6]} z^{[4,6]-1} e^{-z} \mathrm{~d} z .
$$

The expression in (26) is incomplete gamma distribution. Thus, using the neutrosophic calculus and R software, this can be found as

$$
P(Z \leq 6)=[0.554,0.849],
$$

TABLE 1: Performance of likelihood estimates for the distributional parameters of NGD.

\begin{tabular}{lcc}
\hline Sample size & $\mathrm{AB}_{n}$ & $\mathrm{RME}_{n}$ \\
\hline 5 & {$[0.0442,0.07114]$} & {$[3.3052,5.5594]$} \\
15 & {$[0.0058,0.0093]$} & {$[2.7179,4.6696]$} \\
30 & {$[0.0027,0.0044]$} & {$[2.5595,4.4331]$} \\
60 & {$[0.0012,0.0019]$} & {$[2.4752,4.3097]$} \\
\hline
\end{tabular}

which is the required probability that rats would survive no more than 6 weeks.

Moreover, using (5), the average survival time of randomly selected rats can be calculated as

$$
\begin{aligned}
\text { average survival time } & =p_{n} \lambda_{n}=[4,6][1,1] \\
& =[4,6] \text { weeks. }
\end{aligned}
$$

Example 2. In a particular metropolis, the daily usage of water (measured in trillion of litres) roughly follows the NGD with neutrosophic parameters with $p_{n}=[4,5]$ and $\lambda_{n}=[1,1.5]$. What is the probability that the water supply would be insufficient on any given day if the city has a daily capacity of 5 trillion litres of water?

Solution: water supply would be insufficient if consumption is more than daily capacity.

Following (1) reveals that

$$
\begin{aligned}
P[X>5] & =1-P[X \leq 5] \\
& =1-\frac{1}{(\Gamma[4,5])[1,1.5]^{[4,5]}} \int_{0}^{5} z^{[4,5]-1} e^{-z} \mathrm{~d} z .
\end{aligned}
$$

Simplification of (29) using neutrosophic calculus yielded

$$
=[0.50,0.84] \approx[50,84] \% \text {. }
$$

Example 3. The life of a specific type of transistor used in Phillip brand washing machines follows the NGD with a mean of $[4.5,7]$ years and a standard deviation of $[3.7,4.9]$ months. Find the probability that a transistor of this type would survive no more than 1.5 years.

Solution: using the expressions for mean and standard deviation of the NGD, we can write

$$
\begin{aligned}
p_{n} \lambda_{n} & =[4.5,7], \\
\lambda_{n} \sqrt{p_{n}} & =[3.7,4.9] .
\end{aligned}
$$

Simultaneously solving (31) and (32) yielded

$$
\begin{aligned}
& p_{n}=[1.5,2], \\
& \lambda_{n}=[3,3.5] .
\end{aligned}
$$

Thus, the required probability can be calculated as 


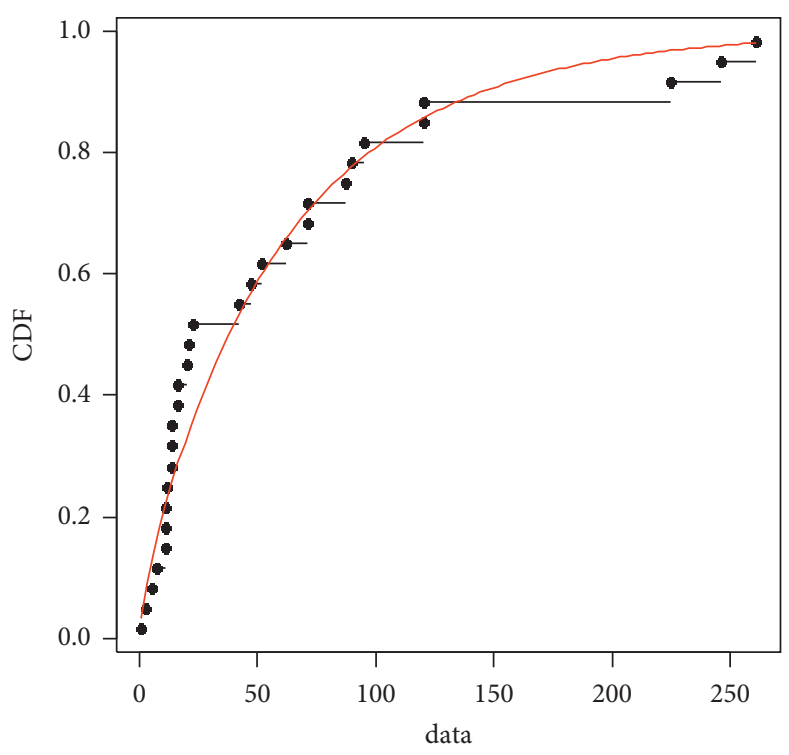

FIgURE 3: Empirical CDF of the air condition system failure data.

$$
\begin{aligned}
P(Z \leq 1.5) & =\frac{1}{(\Gamma[1.5,2])[3,3.5]^{[1.5,2]}} \\
& \int_{0}^{1.5} z^{[1.5,2]-1} e^{-z} \mathrm{~d} z=[0.96,0.97]
\end{aligned}
$$

\section{Real Application}

In this section, the applicability of the suggested NGD has been evaluated by using the dataset given by Linhart and Zucchini [42]. The dataset represents the failure times of the cooling system used in an airplane. Several authors have used this dataset in their study, such as $[43,44]$. The gamma distribution with rate and shape parameters equal to 0.74 and 1, respectively, best describes the failure time data. The appropriateness of gamma distribution with defined parameters can be seen in Figures 3 and 4 .

The CDF plot in Figure 3 indicates how the best-fitted gamma distribution represents the empirical distribution of the observed data. In Figure 4, empirical probabilities calculated from the observed data do not entirely depart from the straight line of the theoretical gamma distribution. Thus, both CDF and PP plots indicate the suitability of the gamma distribution for the observed dataset. Failure measurements are initially crisp values; however, for the sake of illustration, we treat data as uncertain sample values for some air condition systems, as indicated in Table 2.

Table 3 shows that failure times such as $[121,125]$, $[71,74],[11,13]$, and $[52,55]$ are not precisely documented but are available in intervals. Because of ambiguities or uncertainties in the sample, the existing gamma model is not appropriate. On the contrary, the suggested NGD model may simply be used to analyze the given dataset. The descriptive summary of the failure data using the proposed NGD is provided in Table 3.

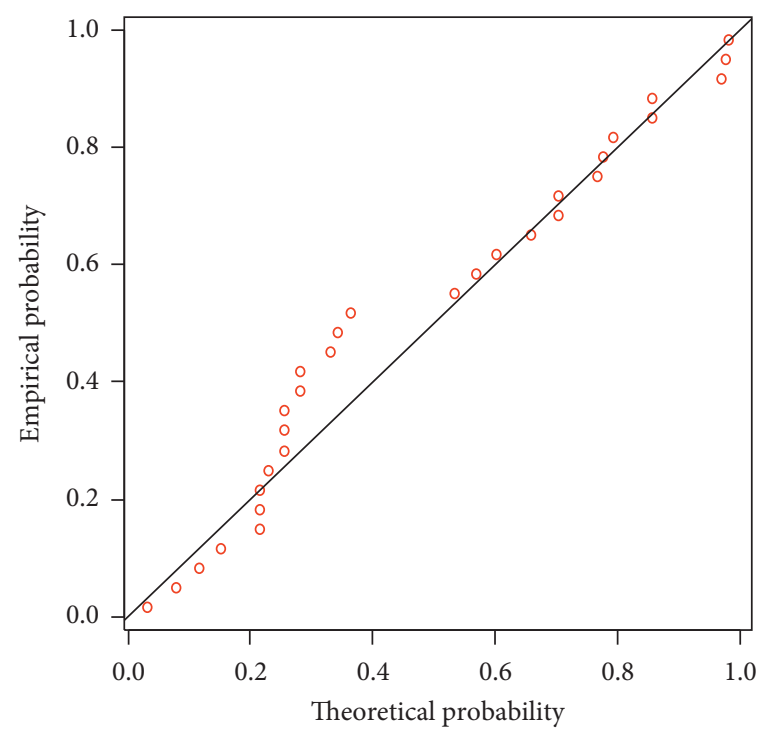

FIgUre 4: Probability plot of the air condition system failure data.

TABLE 2: Failure time data on the air conditioning system.

Failure data on the cooling system

23, 261, 87, 7, [121, 125] 14, 62, 47, 225, [71, 74] 246, 21,42, 20, 5, 12, $120,[11,13] 3,14,71,11,14,11,16,90,1,16,[52,55], 95$

TABLE 3: Descriptive summary of the air conditioning system data using the NGD model.

\begin{tabular}{ll}
\hline \multicolumn{2}{c}{ Descriptive statistics } \\
\hline Mean & {$[1.32,1.36]$} \\
Variance & {$[1.74,1.83]$} \\
Coefficient of skewness & {$[1.71,1.74]$} \\
Coefficient of kurtosis & {$[1.42,1.54]$} \\
\hline
\end{tabular}


It is clearly demonstrated from Table 3 that there are inconsistencies in several of the key numerical statistics of the failure times data due to indeterminacies considered in the observed sample.

\section{Conclusions}

In this work, a neutrosophic extension of the gamma distribution and its applications has been discussed. This extension would open the way for dealing with other classical probability models that are defined for the accurately specified dataset. Using the properties of the neutrosophic calculus, all fundamental properties of the classical gamma model such as moments, shape coefficients, and MGF have been derived. The estimation procedure supported by neutrosophic algebra is developed. Point estimates of the distributional parameters of the proposed NGD have been assessed by the Monte Carlo simulation. Results of the simulation study showed that a large sample size provides reliable estimates of the parameters. Several illustrated examples are discussed to highlight the importance of proposed NGD while handling situations when incomplete or vague information is available about the distributional parameters. A real dataset on failure times of the cooling system with some degree of uncertainty has been analyzed using NGD.

The scope of the present study may be broadened to include other generalized versions of the gamma distribution. Moreover, various properties of the NGD, including testing and estimation methods, based on the neutrosophic logic can be explored in future work. Furthermore, multivariate neutrosophic structure of the proposed model may be worked out.

\section{Data Availability}

Data are included within the manuscript.

\section{Conflicts of Interest}

The authors declare that they have no conflicts of interest.

\section{Acknowledgments}

The authors extend their appreciation to the Deanship of Scientific Research at King Khalid University for funding this work under grant number RGP. 1/26/42, received by Mohammed M. Almazah (https://www.kku.edu.sa). The authors also gratefully acknowledge the Department of Mathematics, Prince Sattam bin Abdulaziz University, for their kind support in this research work.

\section{References}

[1] F. Kamalov and D. Denisov, "Gamma distribution-based sampling for imbalanced data," Knowledge-Based Systems, vol. 207, Article ID 106368, 2020.

[2] B. X. Wang and F. Wu, "Inference on the gamma distribution," Technometrics, vol. 60, no. 2, pp. 235-244, 2017.

[3] N. Chakraborty and T. Mahmood, "Failure rate monitoring in generalized gamma-distributed process," Quality Technology \& Quantitative Management, vol. 18, no. 6, pp. 1-22, 2021.
[4] Y. Zhang, M. Martinez-Garcia, and T. Gordon, "Human response delay estimation and monitoring using gamma distribution analysis," in Proceedings of the Proc. IEEE Int. Conf. Syst. Man, Cybern. SMC, pp. 807-812, IEEE, Miyazaki, Japan, 7 October 2018.

[5] J. del Castillo, J. Daoudi, and I. Serra, "The full tails gamma distribution applied to model extreme values," ASTIN Bulletin, vol. 47, no. 3, pp. 895-917, Sep. 2017.

[6] Z. Ahmad, "The zubair-G family of distributions: properties and applications," Annals of Data Science, vol. 7, no. 2, pp. 195-208, 2018.

[7] C. Cox, H. Chu, M. F. Schneider, and A. Muñoz, "Parametric survival analysis and taxonomy of hazard functions for the generalized gamma distribution," Statistics in Medicine, vol. 26, no. 23, pp. 4352-4374, Oct. 2007.

[8] S. D. Hooper, D. Dalevi, A. Pati, K. Mavromatis, N. N. Ivanova, and N. C. Kyrpides, "Estimating DNA coverage and abundance in metagenomes using a gamma approximation," Bioinformatics, vol. 26, no. 3, pp. 295-301, Feb. 2010.

[9] H. Aksoy, "Use of gamma distribution in hydrological analysis," Turkish Journal of Engineering and Environmental Sciences, vol. 24, no. 6, pp. 419-428, Oct. 2000.

[10] E. R. Ziegel, "Statistical methods for detection and quantification of environmental contamination," Technometrics, vol. 45, no. 2, pp. 176-177, May 2003.

[11] G. J. Husak, J. Michaelsen, and C. Funk, "Use of the gamma distribution to represent monthly rainfall in Africa for drought monitoring applications," International Journal of Climatology, vol. 27, no. 7, pp. 935-944, Jun. 2007.

[12] Z. Chen, W. Yang, Q. Liu, J. Y. Yang, J. Li, and M. Q. Yang, “A new statistical approach to combining p-values using gamma distribution and its application to genome-wide association study," BMC Bioinformatics, vol. 15, no. 17, pp. 1-7, Dec. 2014.

[13] G. Iliopoulos, "Exact confidence intervals for the shape parameter of the gamma distribution," Journal of Statistical Computation and Simulation, vol. 86, no. 8, pp. 1635-1642, Jan. 2016.

[14] K. Krishnamoorthy and L. León-Novelo, "Small sample inference for gamma parameters: one-sample and two-sample problems," Environmetrics, vol. 25, no. 2, pp. 107-126, 2014.

[15] G. Toscani, "Entropy-type inequalities for generalized Gamma densities," Ricerche di Matematica, vol. 70, no. 1, pp. 35-50, 2019.

[16] E. W. Stacy, "A generalization of the gamma distribution," The Annals of Mathematical Statistics, vol. 33, no. 3, pp. 1187-1192, Sep. 1962.

[17] F. Smarandache, A Unifying Field in Logics: Neutrosophic Logic. Neutrosophy, Neutrosophic Set, Neutrosophic Probability, American Research Press, Rehoboth, 1999.

[18] F. Smarandache and S. Pramanik, New Trends in Neutrosophic Theory and Applications, vol. 1, Pons Editions, Brussels, Belgium, 2016.

[19] F. Smarandache, "Neutrosophic set a generalization of the intuitionistic fuzzy sets," International Journal of Pure and Applied Mathematics, vol. 24, no. 3, pp. 287-297, Jun. 2005.

[20] M. Ali, L. Q. Dat, L. H. Son, and F. Smarandache, "Interval complex neutrosophic set: formulation and applications in decision-making," International Journal of Fuzzy Systems, vol. 20, no. 3, pp. 986-999, Mar. 2018.

[21] A. A. Salama and S. A. Alblowi, "Generalized neutrosophic set and generalized neutrosophic spaces," J. Computer Sci. Engineering, vol. 2, no. 7, pp. 129-132, 2012. 
[22] F. Smarandache and S. Pramanik, New Trends in Neutrosophic Theory and Applications, vol. 2, Pons Editions, Brussels, Belgium, 2018.

[23] M. Abdel-Basset, Y. Zhou, M. Mohamed, and V. Chang, "A group decision making framework based on neutrosophic VIKOR approach for e-government website evaluation," Journal of Intelligent and Fuzzy Systems, vol. 34, no. 6, pp. 4213-4224, Jan 2018.

[24] F. Smarandache, Neutrosophical Statistics, Sitech \& Education publishing, Craiova, Romania, 2014.

[25] F. Smarandache, "Neutrosophy and neutrosophic logic," in Proceedings of the First International Conference on Neutrosophy, Neutrosophic Logic, Set, Probability, and Statistics, University of New Mexico, Gallup, NM 87301, USA, 1 December 2002.

[26] S. K. Patro and F. Smarandache, "The neutrosophic statistics distribution, more Problems, more solutions," Neutrosophic Sets and Systems, vol. 12, 2016.

[27] F. Smarandache, Introduction to Neutrosophic Measure, Integral, Probability, Sitech Education publisher, 2015.

[28] A. A. Salama, F. Smarandache, and S. A. Alblowi, New Neutrosophic Crisp Topological Concepts, Neutrosophic Sets and Systems, 2014.

[29] Z. Khan, M. Gulistan, W. Chammam, S. Kadry, and Y. Nam, "A new dispersion control chart for handling the neutrosophic data," IEEE Access, vol. 8, pp. 96006-96015, 2020.

[30] Z. Khan, M. Gulistan, R. Hashim, N. Yaqoob, and W. Chammam, "Design of S-control chart for neutrosophic data: an application to manufacturing industry," Journal of Intelligent and Fuzzy Systems, vol. 38, no. 4, pp. 4743-4751, 2020.

[31] Z. Khan, M. Gulistan, N. Kausar, and C. Park, "Neutrosophic Rayleigh model with some basic characteristics and engineering applications," IEEE Access, vol. 9, pp. 71277-71283, 2021.

[32] M. Aslam, N. Khan, and M. Khan, "Monitoring the variability in the process using neutrosophic statistical interval method," Symmetry, vol. 10, no. 11, p. 562, Nov. 2018.

[33] F. Smarandache and H. E. Khalid, Neutrosophic Precalculus and Neutrosophic Calculus, University of New Mexico, Infinite Study, 2018.

[34] R. Alhabib, M. M. Ranna, H. Farah, and A. A. Salama, "Some neutrosophic probability distributions," Neutrosophic Sets and Systems, vol. 38, pp. 30-38, 2018.

[35] W.-Q. Duan, Z. Khan, M. Gulistan, and A. Khurshid, "Neutrosophic exponential distribution: modeling and applications for complex data analysis," Complexity, vol. 2021, Article ID 5970613, 8 pages, 2021.

[36] R. A. K. Sherwani, M. Aslam, M. A. Raza, M. Farooq, M. Abid, and M. Tahir, "Neutrosophic normal probability distributionA spine of parametric neutrosophic statistical tests: properties and applications," in Neutrosophic Operational Research, pp. 153-169, Springer, Berlin, Germany, 2021.

[37] R. A. K. Sherwan, M. Naeem, M. Aslam, M. A. Raza, M. Abid, and S. Abbas, "Neutrosophic beta distribution with properties and applications," Neutrosophic Sets Syst, vol. 41, pp. 209-214, 2021.

[38] A. M. Almarashi and M. Aslam, "Process monitoring for gamma distributed product under neutrosophic statistics using resampling scheme," Jurnal Matematika, vol. 2021, Article ID 6635846, 12 pages, 2021.

[39] R. A. K. Sherwani, H. Shakeel, M. Saleem, W. B. Awan, M. Aslam, and M. Farooq, "A new neutrosophic sign test: an application to COVID-19 data," PLoS One, vol. 16, no. 8, Article ID e0255671, 2021.

[40] M. Aslam, "Neutrosophic statistical test for counts in climatology," Scientific Reports, vol. 11, no. 1, pp. 1-5, Sep. 2021.

[41] F. Shah, Z. Khan, M. Aslam, and S. Kadry, "Statistical development of the VSQ-control chart for extreme data with an application to the carbon fiber industry," Mathematical Problems in Engineering, vol. 2021, Article ID 9766986, 11 pages, Jul. 2021.

[42] H. Linhart and W. Zucchini, Model Selection, John Wiley \& Sons, New York, 1986.

[43] T. M. Magalhães, Y. M. Gómez, D. I. Gallardo, and O. Venegas, "Bias reduction for the marshall-olkin extended family of distributions with application to an airplane air conditioning system and precipitation data," Symmetry, vol. 12 , no. 5, p. 851, May 2020.

[44] M. S. Khan and R. King, "New generalized inverse Weibull distribution for lifetime modeling," Communications for Statistical Applications and Methods, vol. 23, no. 2, pp. 147-161, 2016. 